\title{
Cycloaddition of $\Delta^{2}$-Thiazolines and Acyl Ketenes under Acidic Conditions results in Bicyclic 1,3- Oxazines not 6-Acylpenams as earlier reported.
}

Nils Pemberton ${ }^{\dagger}$, Hans Emtenäs ${ }^{\dagger}$, Dan Boström ${ }^{\ddagger}$, Peter J. Domaille, William A. Greenberg, Michael D. Levin, Zuolin Zhu ${ }^{\S}$, and Fredrik Almqvist**

Organic Chemistry and Inorganic Chemistry, Umeå University, S-901 87 Umeå, Sweden and Diversa Corporation, 4955 Directors Place, San Diego, CA 92121 USA

fredrik.almqvist@chem.umu.se

\section{Supporting Information}

\section{Table of contents}

S1-6: experimental procedures

S7-10: $\mathrm{C}^{13}-\mathrm{C}^{13}$ Inadequate data

General. All reactions were carried out under an inert atmosphere with dry solvents under anhydrous conditions, unless otherwise stated. $\mathrm{CH}_{2} \mathrm{Cl}_{2}$ were distilled from calcium hydride and THF from potassium, respectively. Benzene and toluene were distilled from sodium. TLC was performed on Silica Gel $60 \mathrm{~F}_{254}$ (Merck) with detection by UV light and staining with a solution of anisaldehyde $(26 \mathrm{ml})$, glacial acetic acid $(11 \mathrm{ml})$ and concentrated sulphuric acid $(35 \mathrm{ml})$ in $95 \%$ ethanol $(960 \mathrm{ml})$. Flash column chromatography (eluents given in brackets) was performed on silica gel (Matrex, $60 \AA$, 35-70 $\mu \mathrm{m}$, Grace Amicon). The ${ }^{1} \mathrm{H}$ and ${ }^{13} \mathrm{C}$ NMR spectra were recorded on a Bruker DRX-400 for solutions in $\mathrm{CDCl}_{3}$ [residual $\mathrm{CHCl}_{3}\left(\delta_{\mathrm{H}} 7.26 \mathrm{ppm}\right)$ or $\mathrm{CDCl}_{3}\left(\delta_{\mathrm{C}} 77.0 \mathrm{ppm}\right)$ as internal standard] or $\mathrm{CD}_{3} \mathrm{OD}$ [residual $\mathrm{CD}_{3} \mathrm{OD}\left(\delta_{\mathrm{H}} 4.87 \mathrm{ppm}\right)$ at $298 \mathrm{~K}$. First-order chemical shifts and coupling constants were obtained from one-dimensional spectra and proton resonances were assigned from appropriate combinations of COSY. IR spectra were recorded on an ATI Mattson Genesis Series FTIR ${ }^{\mathrm{TM}}$ spectrometer. Optical rotations were measured with a Perkin-Elmer 343 polarimeter. Positive fast atom bombardment mass spectra (FABMS) were recorded on a JEOL SX102 A mass spectrometer. Ions for 
FABMS were produced by a beam of Xenon atoms $(6 \mathrm{keV})$ from a matrix of glycerol and thioglycerol.

\section{(3R,9R)-5-Oxo-7-phenyl-2,3-dihydro-5H-thiazolo[2,3-b][1,3]oxazine-3-carboxylic} acid methyl ester (3a).

Meldrum's acid derivative 1a (1027 mg, $4.14 \mathrm{mmol})$ and thiazoline $2(394 \mathrm{mg}, 2.72$ mmol) were dissolved in dry benzene $(44 \mathrm{ml})$ and cooled to $5{ }^{\circ} \mathrm{C}$. Dry $\mathrm{HCl}$ gas was bubbled through the mixture for 10 minutes. The resulting turbid mixture was heated for 1.5 hours at $79{ }^{\circ} \mathrm{C}$ and then cooled to room temperature. The resulting mixture was diluted with ethyl acetate and washed with ice cooled water and brine. The water phase was extracted twice with $\mathrm{CH}_{2} \mathrm{Cl}_{2}$ and the combined organic extracts where dried with $\mathrm{Na}_{2} \mathrm{SO}_{4}$, filtered and evaporated. Flash chromatography (heptane:ethyl acetate 1:1 -> 3:7) gave 3a ( $643 \mathrm{mg}, 81 \%$ ): $[\alpha]_{\mathrm{D}}{ }^{20} 38^{\circ}$ (c 2.60, $\mathrm{CHCl}_{3}$ ); IR $\lambda$ 3062, 3004, 2952, 1739, 1658, 1604, $1387 \mathrm{~cm}^{-1}:{ }^{1} \mathrm{H}$ NMR (400 MHz, $\left.\mathrm{CDCl}_{3}\right) \delta 7.70(\mathrm{~m}, 2 \mathrm{H}), 7.40-7.51(\mathrm{~m}, 3 \mathrm{H}), 6.68$ (s, 1H), $6.00(\mathrm{~s}, 1 \mathrm{H}), 5.35(\mathrm{~d}, 1 \mathrm{H}, J=6.40 \mathrm{~Hz}), 3.81(\mathrm{~s}, 1 \mathrm{H}), 3.55\left(\mathrm{dd}, 1 \mathrm{H}, J_{I}=11.25 \mathrm{~Hz}\right.$, $\left.J_{2}=6.40 \mathrm{~Hz}\right), 3.30(\mathrm{~d}, 1 \mathrm{H}, J=11.25 \mathrm{~Hz}):{ }^{13} \mathrm{C} \mathrm{NMR}\left(100 \mathrm{MHz}, \mathrm{CDCl}_{3}\right) \delta 168.7,164.3$, 161.5, 131.7, 130.8, 128.8, 126.6, 97.7, 94.4, 60.9, 53.1, 32.1; HRMS (EI+) Calcd. for $\mathrm{C}_{14} \mathrm{H}_{13} \mathrm{NO}_{4} \mathrm{~S}: 291.0565$ Observed 291.0567

\section{(3R,9R)-7-Naphthalen-1-yl-5-oxo-2,3-dihydro-5H-thiazolo[2,3-b][1,3]oxazine-3-}

carboxylic acid methyl ester (3b). Meldrum's acid derivative $1 \mathbf{b}(630 \mathrm{mg}, 2.11 \mathrm{mmol})$ and thiazoline 2 (185 $\mathrm{mg}, 1.27 \mathrm{mmol})$ were dissolved in dry benzene $(28 \mathrm{ml})$ and cooled to $5{ }^{\circ} \mathrm{C}$. Dry $\mathrm{HCl}$ gas was bubbled through the mixture for 10 minutes. The resulting turbid mixture was heated for 1.5 hours at $79{ }^{\circ} \mathrm{C}$ and then cooled to room temperature. The resulting mixture was diluted with ethyl acetate and washed with ice cooled water and brine. The water phase was extracted twice with $\mathrm{CH}_{2} \mathrm{Cl}_{2}$ and the combined organic extracts where dried with $\mathrm{Na}_{2} \mathrm{SO}_{4}$, filtered and evaporated. Flash chromatography (heptane:ethyl acetate 1:1 -> 3:7) gave $\mathbf{3 b}\left(403 \mathrm{mg}, 93 \%\right.$ ) as a white foam: $[\alpha]_{\mathrm{D}}{ }^{20} 2.7^{\circ}$ (c 1.77, $\mathrm{CHCl}_{3}$ ); IR $\lambda 1739,1664,1508,1388,1211,977,773 \mathrm{~cm}^{-1} ;{ }^{1} \mathrm{H}$ NMR $(400 \mathrm{MHz}$, $\left.\mathrm{CDCl}_{3}\right) \delta 8.14(\mathrm{~d}, 1 \mathrm{H}, J=8.23 \mathrm{~Hz}), 7.96(\mathrm{~d}, 1 \mathrm{H}, J=8.23 \mathrm{~Hz}), 7.89(\mathrm{~m}, 1 \mathrm{H}), 7.66(\mathrm{dd}$, 
$\left.1 \mathrm{H}, J_{1}=7.23 \mathrm{~Hz}, J_{2}=1.19 \mathrm{~Hz}\right), 7.46-7.59(\mathrm{~m}, 3 \mathrm{H}), 6.93(\mathrm{~s}, 1 \mathrm{H}), 5.89(\mathrm{~s}, 1 \mathrm{H}), 5.43(\mathrm{~d}, 1 \mathrm{H}$, $J=6.31 \mathrm{~Hz}), 3.84(\mathrm{~s}, 3 \mathrm{H}), 3.60\left(\mathrm{dd}, 1 \mathrm{H}, J_{1}=11.25 \mathrm{~Hz}, J_{2}=6.40 \mathrm{~Hz}\right), 3.32(\mathrm{~d}, 1 \mathrm{H}, J=$ 11.25); ${ }^{13} \mathrm{C}$ NMR (100 MHz, $\left.\mathrm{CDCl}_{3}\right) \delta 169.7,165.7,161.2,133.7,131.7,130.6,129.7$, 128.2, 127.3, 126.4, 124.9, 124.8, 103.4, 94.4, 60.9, 53.2, 32.1; HRMS (FAB+) Calcd. for $\mathrm{C}_{18} \mathrm{H}_{16} \mathrm{NO}_{4} \mathrm{~S}: 342.0800$ Observed 342.0803.

$(3 R, 9 R)-7-N a p h t h a l e n-1-y l m e t h y l-5-0 x 0-2,3-d i h y d r o-5 H$-thiazolo[2,3-b][1,3] oxazine3-carboxylic acid methyl ester (3c). Meldrum's acid derivative 1c (1250 mg, 4.00 mmol) and thiazoline 2 (440 mg, $3.03 \mathrm{mmol}$ ) were dissolved in dry benzene (55 $\mathrm{ml}$ ) and cooled to $5{ }^{\circ} \mathrm{C}$. Dry $\mathrm{HCl}$ gas was bubbled through the mixture for 15 minutes. The resulting turbid mixture was heated for 3 hours at $79{ }^{\circ} \mathrm{C}$ and then cooled to room temperature. The resulting mixture was diluted with ethyl acetate and washed with ice cooled water and brine. The water phase was extracted twice with $\mathrm{CH}_{2} \mathrm{Cl}_{2}$ and the combined organic extracts where dried with $\mathrm{Na}_{2} \mathrm{SO}_{4}$, filtered and evaporated. Flash chromatography (heptane:ethyl acetate 1:1 -> 3:7) gave 3c (660 mg, $62 \%$ ) as a white foam: $[\alpha]_{\mathrm{D}}{ }^{20}-3.75^{\circ}\left(\mathrm{c} 0.40, \mathrm{CHCl}_{3}\right)$; IR $\lambda$ 3008, 2952, 1743, 1664, 1396, 1215, 968, 752 $\mathrm{cm}^{-1} ;{ }^{1} \mathrm{H}$ NMR $\left(400 \mathrm{MHz}, \mathrm{CDCl}_{3}\right) \delta 7.88(\mathrm{~m}, 2 \mathrm{H}), 7.81,(\mathrm{~d}, 1 \mathrm{H}, J=8.14 \mathrm{~Hz}), 7.51(\mathrm{~m}$, 2H), $7.43(\mathrm{~m}, 1 \mathrm{H}), 7.36(\mathrm{~d}, 1 \mathrm{H}, J=6.77 \mathrm{~Hz}), 6.55(\mathrm{~s}, 1 \mathrm{H}), 5.27(\mathrm{~d}, 1 \mathrm{H}, J=6.31 \mathrm{~Hz})$, $5.10(\mathrm{~s}, 1 \mathrm{H}), 4.00(\mathrm{~s}, 2 \mathrm{H}), 3.75(\mathrm{~s}, 3 \mathrm{H}), 3.49\left(\mathrm{dd}, 1 \mathrm{H}, J_{1}=11.16 \mathrm{~Hz}, J_{2}=6.40 \mathrm{~Hz}\right), 3.23$ $(\mathrm{d}, 1 \mathrm{H}, J=11.16 \mathrm{~Hz}) ;{ }^{13} \mathrm{C} \mathrm{NMR}\left(100 \mathrm{MHz}, \mathrm{CDCl}_{3}\right) \delta 169.5,168.9,160.7,133.9$, $131.8,130.5,128.8,128.4,128.1,126.5,125.9,125.4,123.4,101.1,94.0,60.7,53.0$, 36.7, 31.9; HRMS (FAB+) Calcd. for C19H18NO4S 356.0957 Observed 356.0960.

\section{$(3 R, 9 R)$-7-Naphthalen-2-ylmethyl-5-oxo-2,3-dihydro-5H-thiazolo[2,3-b][1,3] oxazine-}

3-carboxylic acid methyl ester (3d). Meldrum's acid derivative 1d (1263 mg, 4.04 $\mathrm{mmol}$ ) and thiazoline 2 (392 $\mathrm{mg}, 2.70 \mathrm{mmol}$ ) were dissolved in dry benzene (60 $\mathrm{ml})$ and cooled to $5{ }^{\circ} \mathrm{C}$. Dry $\mathrm{HCl}$ gas was bubbled through the mixture for 10 minutes. The resulting turbid mixture was heated for 2.5 hours at $79{ }^{\circ} \mathrm{C}$ and then cooled to room temperature. The resulting mixture was diluted with ethyl acetate and washed with ice cooled water and brine. The water phase was extracted twice with $\mathrm{CH}_{2} \mathrm{Cl}_{2}$ and the 
combined organic extracts where dried with $\mathrm{Na}_{2} \mathrm{SO}_{4}$, filtered and evaporated. Flash chromatography (heptane:ethyl acetate 1:1 -> 3:7) gave 3d (622 mg, $65 \%$ ): $[\alpha]_{\mathrm{D}}^{20} 3.3^{\circ}$ (c 2.96, $\left.\mathrm{CHCl}_{3}\right)$; IR $\lambda$ 3049, 3010, 1741, 1666, 1392, 1209, 964, 813, $746 \mathrm{~cm}^{-1} ;{ }^{1} \mathrm{H}$ NMR (400 MHz, $\left.\mathrm{CDCl}_{3}\right) \delta 7.80(\mathrm{~m}, 3 \mathrm{H}), 7.66(\mathrm{~s}, 1 \mathrm{H}), 7.48(\mathrm{~m}, 2 \mathrm{H}), 7.32\left(\mathrm{dd}, 1 \mathrm{H}, J_{l}=8.51\right.$ $\left.\mathrm{Hz}, J_{2}=1.37 \mathrm{~Hz}\right), 6.50(\mathrm{~s}, 1 \mathrm{H}), 5.31(\mathrm{~s}, 1 \mathrm{H}), 5.28(\mathrm{~d}, 1 \mathrm{H}, J=6.31 \mathrm{~Hz}), 3.74(\mathrm{~s}, 3 \mathrm{H}), 3.69$ $(\mathrm{s}, 2 \mathrm{H}), 3.44\left(\mathrm{dd}, 1 \mathrm{H}, J_{1}=11.16 \mathrm{~Hz}, J_{2}=6.50 \mathrm{~Hz}\right), 3.20(\mathrm{~d}, 1 \mathrm{H}, J=11.16 \mathrm{~Hz}) ;{ }^{13} \mathrm{C}$ NMR (100 MHz, CDCl3) $\delta 169.5,168.9,160.7,133.3,132.4,130.1,128.4,127.9,127.6$, 127.5, 126.9, 126.2, 125.9, 100.9, 94.0, 60.6, 52.9, 39.6, 31.7; HRMS (CI+) Calcd. for $\mathrm{C}_{19} \mathrm{H}_{18} \mathrm{NO}_{4} \mathrm{~S}: 356.0957$ Observed 356.0956.

\section{(3R,9R)-7-Cyclohexyl-5-oxo-2,3-dihydro-5H-thiazolo[2,3-b][1,3]oxazine-3-carboxylic} acid methyl ester (3e). Meldrum's acid derivative 1e (1210 mg, $4.76 \mathrm{mmol})$ and thiazoline 2 (435 mg, $3.00 \mathrm{mmol}$ ) were dissolved in dry benzene $(50 \mathrm{ml})$ and cooled to 5 ${ }^{\circ} \mathrm{C}$. Dry $\mathrm{HCl}$ gas was bubbled through the mixture for 12 minutes. The resulting turbid mixture was heated for 2 hours at $79^{\circ} \mathrm{C}$ and then cooled to room temperature. The resulting mixture was diluted with ethyl acetate and washed with ice cooled water and brine. The water phase was extracted twice with $\mathrm{CH}_{2} \mathrm{Cl}_{2}$ and the combined organic extracts where dried with $\mathrm{Na}_{2} \mathrm{SO}_{4}$, filtered and evaporated. Flash chromatography (heptane:ethyl acetate $1: 1 \rightarrow 3: 7$ ) of the residue and then chromatotrone $2 \mathrm{~mm}$ (heptane:ethyl acetate 1:1) gave 3e ( $644 \mathrm{mg}, 72 \%$ ): $[\alpha]_{\mathrm{D}}^{20} 7.4^{\circ}\left(\mathrm{c} 2.28, \mathrm{CHCl}_{3}\right)$; IR $\lambda 2929,2852,1736,1657,1610,1404,1296,1223,1005,960,933,804,756 \mathrm{~cm}^{-1} ;{ }^{1} \mathrm{H}$ NMR (400 MHz, CDCl3) $\delta 6.45(\mathrm{~s}, 1 \mathrm{H}), 5.30(\mathrm{~s}, 1 \mathrm{H}), 5.28(\mathrm{~d}, 1 \mathrm{H}, J=6.31 \mathrm{~Hz}), 3.77(\mathrm{~s}$, $3 \mathrm{H}), 3.48\left(\mathrm{dd}, 1 \mathrm{H}, J_{1}=11.25 \mathrm{~Hz}, J_{2}=6.50 \mathrm{~Hz}\right), 3.23(\mathrm{~d}, 1 \mathrm{H}, J=11.25), 2.14(\mathrm{~m}, 1 \mathrm{H})$, $1.82(\mathrm{~m}, 4 \mathrm{H}), 1.69(\mathrm{~m}, 1 \mathrm{H}), 1.11-1.36(\mathrm{~m}, 5 \mathrm{H}) ;{ }^{13} \mathrm{C}$ NMR $\left(100 \mathrm{MHz}, \mathrm{CDCl}_{3}\right) \delta$ 174.4, 169.8, 161.3, 97.9, 93.8, 60.7, 53.0, 41.7, 31.9, 30.1, 29.8, 35.8, 25.7, 25.6; HRMS (EI+) Calcd. for $\mathrm{C}_{14} \mathrm{H}_{19} \mathrm{NO}_{4} \mathrm{~S}: 297.1035$ Observed 297.1035. 
(3R,9R)-7-Pentyl-5-oxo-2,3-dihydro-5H-thiazolo[2,3-b][1,3]oxazine-3-carboxylic acid methyl ester (3f).

Meldrum's acid derivative 1f $(1133 \mathrm{mg}, 4.67 \mathrm{mmol})$ and thiazoline $2(443 \mathrm{mg}, 3.05$ mmol) were dissolved in dry benzene $(50 \mathrm{ml})$ and cooled to $5{ }^{\circ} \mathrm{C}$. Dry $\mathrm{HCl}$ gas was bubbled through the mixture for 12 minutes. The resulting turbid mixture was heated for 2 hours at $79^{\circ} \mathrm{C}$ and then cooled to room temperature. The resulting mixture was diluted with ethyl acetate and washed with ice cooled water and brine. The water phase was extracted twice with $\mathrm{CH}_{2} \mathrm{Cl}_{2}$ and the combined organic extracts where dried with $\mathrm{Na}_{2} \mathrm{SO}_{4}$, filtered and evaporated. Flash chromatography (heptane:ethyl acetate 1:1 $>>3: 7$ ) of the residue and then chromatotrone $2 \mathrm{~mm}$ (heptane:ethyl acetate 1:1) gave 3f ( $696 \mathrm{mg}, 80$ $\%$ ): $[\alpha]_{\mathrm{D}}{ }^{20} 7.3^{\circ}$ (c 1.94, $\mathrm{CHCl}_{3}$ ); IR $\lambda 2954,2931,1743,1670,1614,1394,1296,1211$, $968,810 \mathrm{~cm}^{-1}:{ }^{1} \mathrm{H}$ NMR $\left(400 \mathrm{MHz}, \mathrm{CDCl}_{3}\right) \delta 6.48(\mathrm{~s}, 1 \mathrm{H}), 5.31(\mathrm{~s}, 1 \mathrm{H}), 5.28(\mathrm{~d}, 1 \mathrm{H}, J=$ $6.40 \mathrm{~Hz}), 3.77(\mathrm{~s}, 3 \mathrm{H}), 3.47\left(\mathrm{dd}, 1 \mathrm{H}, J_{1}=11.25 \mathrm{~Hz}, J_{2}=6.40 \mathrm{~Hz}\right), 3.22(\mathrm{~d}, 1 \mathrm{H}, J=11.25$ $\mathrm{Hz}), 2.23(\mathrm{~m}, 2 \mathrm{H}), 1.53$ (p, 2H, $J=7.50 \mathrm{~Hz}), 1.30(\mathrm{~m}, 4 \mathrm{H}), 0.88(\mathrm{t}, 3 \mathrm{H}, J=7.20 \mathrm{~Hz})$ : ${ }^{13} \mathrm{C}$ NMR $\left(100 \mathrm{MHz}, \mathrm{CDCl}_{3}\right) \delta$ 170.6, 169.7, 161.0, 99.7, 93.7, 60.7, 53.0, 33.1, 31.9, 31.1, 25.8, 22.2, 13.9; HRMS (EI+) Calcd. for $\mathrm{C}_{13} \mathrm{H}_{19} \mathrm{NO}_{4} \mathrm{~S}: 285.1035$ Observed 285.1033

\section{(3R,9R)-7-Methyl-5-oxo-2,3-dihydro-5H-thiazolo[2,3-b][1,3]oxazine-3-carboxylic acid methyl ester (3g).}

Meldrum's acid derivative $\mathbf{1 g}(738 \mathrm{mg}, 3.96 \mathrm{mmol})$ and thiazoline 2 (377 mg, $2.60 \mathrm{mmol})$ were dissolved in dry benzene $(41 \mathrm{ml})$ and cooled to $5{ }^{\circ} \mathrm{C}$. Dry $\mathrm{HCl}$ gas was bubbled through the mixture for 10 minutes. The resulting turbid mixture was heated for 2 hours at $79^{\circ} \mathrm{C}$ and then cooled to room temperature. The resulting mixture was diluted with ethyl acetate and washed with ice cooled water and brine. The water phase was extracted twice with $\mathrm{CH}_{2} \mathrm{Cl}_{2}$ and the combined organic extracts where dried with $\mathrm{Na}_{2} \mathrm{SO}_{4}$, filtered and evaporated. Flash chromatography (heptane:ethyl acetate 1:1 -> 3:7) gave 3g ( 227 mg, $38 \%$ ): $[\alpha]_{\mathrm{D}}{ }^{20} 12^{\circ}$ (c 2.76, $\mathrm{CHCl}_{3}$ ); IR $\lambda$ 3008, 2953, 1739, 1666, 1396, 1356, 1209 , 1157, 966, 812, $748 \mathrm{~cm}^{-1}:{ }^{1} \mathrm{H}$ NMR (400 MHz, $\left.\mathrm{CDCl}_{3}\right) \delta 6.49(\mathrm{~s}, 1 \mathrm{H}), 5.30(\mathrm{~s}, 1 \mathrm{H}), 5.26$ $(\mathrm{d}, 1 \mathrm{H} J=6.31 \mathrm{~Hz}), 3.75(\mathrm{~s}, 3 \mathrm{H}), 3.45\left(\mathrm{dd}, 1 \mathrm{H}, J_{l}=11.34 \mathrm{~Hz}, J_{2}=6.31 \mathrm{~Hz}\right), 3.21(\mathrm{~d}, 1 \mathrm{H}$, 
$J=11.34 \mathrm{~Hz}), 1.98(\mathrm{~s}, 3 \mathrm{H}):{ }^{13} \mathrm{C} \mathrm{NMR}\left(100 \mathrm{MHz}, \mathrm{CDCl}_{3}\right) \delta 169.6,166.9,160.8,100.4$, 93.6, 60.7, 53.0, 31.8, 19.3; HRMS (EI+) Calcd. for $\mathrm{C}_{9} \mathrm{H}_{11} \mathrm{NO}_{4} \mathrm{~S}$ : 229.0397 Observed 229.0407. 
${ }^{13} \mathrm{C}$ assignments and connectivity for Compound $\mathbf{3}$ where $\mathrm{R}=$ phenyl, ethyl ester

\begin{tabular}{|c|c|c|}
\hline Atom & Chemical shift (ppm) & $\underline{J}_{\mathrm{C}-\mathrm{C}}(\mathrm{Hz})$ \\
\hline C2 & 31.7 & $34.2(2,3)$ \\
\hline C3 & 60.6 & $34.2(3,2) ; 63.7$ (3, COOEt) \\
\hline C5 & 160.9 & $66.9(5,6)$ \\
\hline C6 & 97.3 & $66.9(6,5) ; 73.1(6,7)$ \\
\hline $\mathrm{C7}$ & 163.7 & $73.1(7,6) ; 64.4$ (7, quaternary aromatic) \\
\hline C9 & 94.1 & none \\
\hline $\mathrm{CH}_{3}$ (ester) & 13.8 & $38.7\left(\mathrm{CH}_{3}, \mathrm{CH}_{2}\right)$ \\
\hline $\mathrm{CH}_{2}$ (ester) & 61.7 & $38.7\left(\mathrm{CH}_{2}, \mathrm{CH}_{3}\right)$ \\
\hline COOEt & 168.7 & 63.7 (COOEt, C3) \\
\hline
\end{tabular}



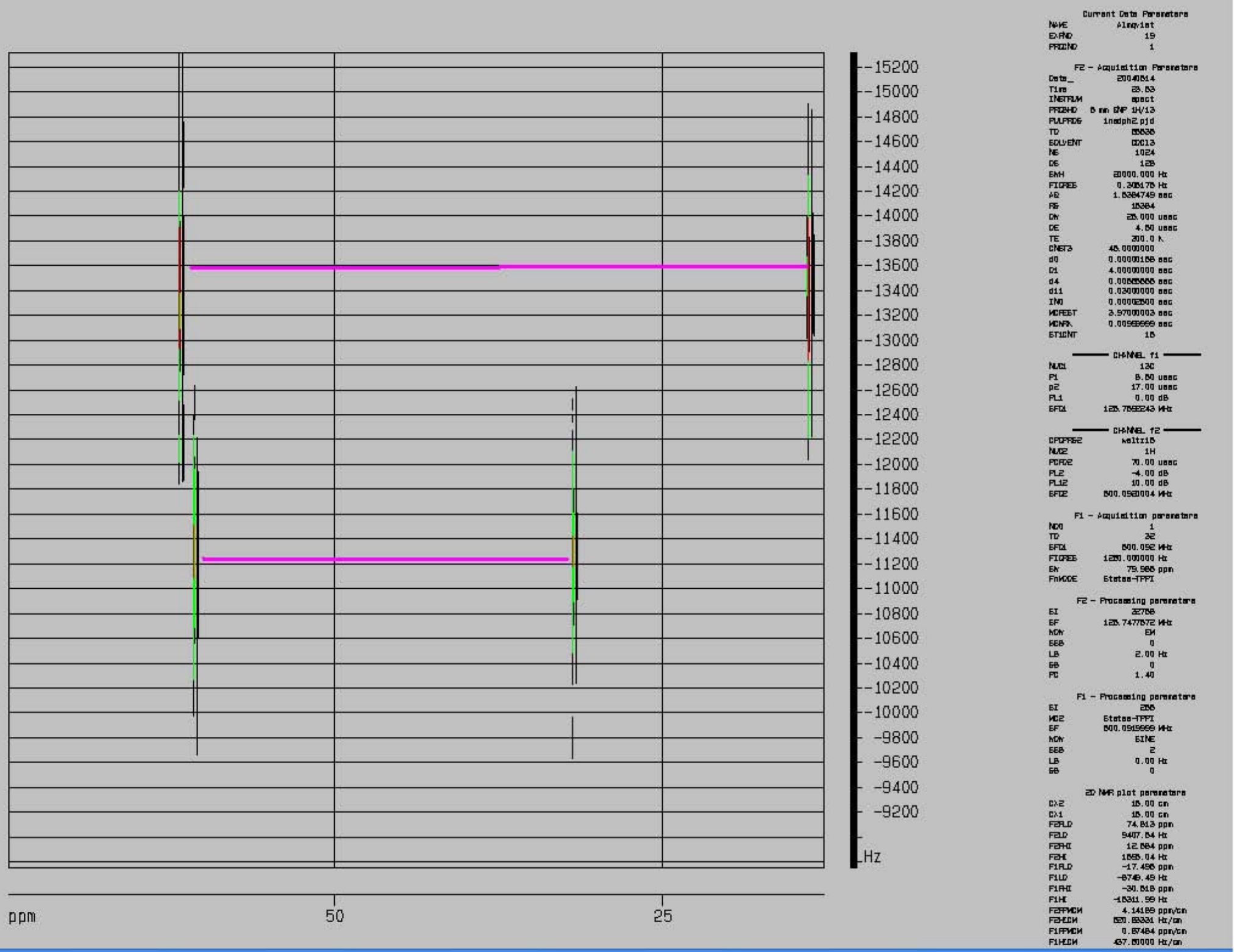

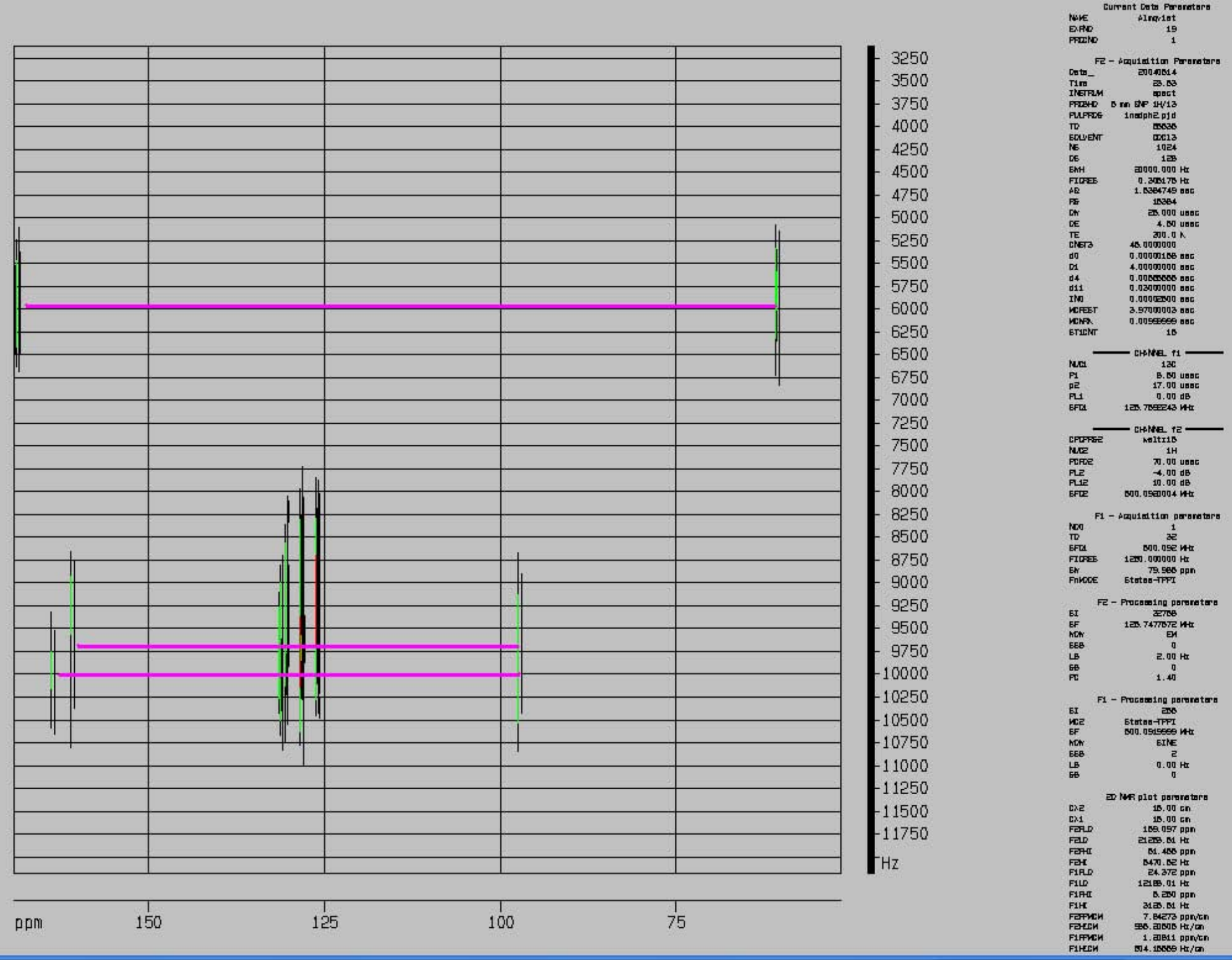